\title{
Amyloid-beta modulates the association between neurofilament light chain and brain atrophy in Alzheimer's disease
}

\author{
Min Su Kang $\mathbb{1}^{1,2,3} \cdot$ Arturo Aliaga Aliaga ${ }^{1,2,3} \cdot$ Monica Shin ${ }^{1,2} \cdot$ Sulantha Mathotaarachchi ${ }^{1,2}$. \\ Andrea L. Benedet $\mathbb{B}^{1,2} \cdot$ Tharick A. Pascoall ${ }^{1,2} \cdot$ Joseph Therriault $\mathbb{D}^{1,2} \cdot$ Mira Chamoun ${ }^{1,2} \cdot$ Melissa Savard $^{1,2}$. \\ Gabriel A. Devenyi $\mathbb{1}^{2,4} \cdot$ Axel Mathieu $^{2} \cdot$ M. Mallar Chakravarty ${ }^{2,4,5}$. Åsa Sandelius ${ }^{6}$. Kaj Blennow $\mathbb{D}^{6,7}$. \\ Henrik Zetterberg ${ }^{6,7,8,9} \cdot$ Jean-Paul Soucy ${ }^{3} \cdot$ A. Claudio Cuello ${ }^{10} \cdot$ Gassan Massarweh $^{3} \cdot$ Serge Gauthier $^{1,2,3}$. \\ Pedro Rosa-Neto $\mathbb{1}^{1,2,3,4} \cdot$ Alzheimer's Disease Neuroimaging Initiative
}

Received: 18 July 2019 / Revised: 3 June 2020 / Accepted: 9 June 2020 / Published online: 26 June 2020

(c) The Author(s) 2020. This article is published with open access

\begin{abstract}
Neurofilament light chain (NFL) measurement has been gaining strong support as a clinically useful neuronal injury biomarker for various neurodegenerative conditions. However, in Alzheimer's disease (AD), its reflection on regional neuronal injury in the context of amyloid pathology remains unclear. This study included 83 cognitively normal (CN), 160 mild cognitive impairment (MCI), and $73 \mathrm{AD}$ subjects who were further classified based on amyloid-beta (A $\beta)$ status as positive or negative $(\mathrm{A} \beta+\mathrm{vs} A \beta-)$. In addition, 13 rats (5 wild type and 8 McGill-R-Thy1-APP transgenic $(\mathrm{Tg})$ ) were examined. In the clinical study, reduced precuneus/posterior cingulate cortex and hippocampal grey matter density were significantly associated with increased NFL concentrations in cerebrospinal fluid (CSF) or plasma in MCI A $\beta+$ and $\mathrm{AD} A \beta+$. Moreover, $\mathrm{AD} A \beta+$ showed a significant association between the reduced grey matter density in the ADvulnerable regions and increased NFL concentrations in CSF or plasma. Congruently, Tg rats recapitulated and validated the association between CSF NFL and grey matter density in the parietotemporal cortex, entorhinal cortex, and hippocampus in the presence of amyloid pathology. In conclusion, reduced grey matter density and elevated NFL concentrations in CSF and plasma are associated in $\mathrm{AD}$-vulnerable regions in the presence of amyloid positivity in the AD clinical spectrum and amyloid Tg rat model. These findings further support the NFL as a neuronal injury biomarker in the research framework of $\mathrm{AD}$ biomarker classification and for the evaluation of therapeutic efficacy in clinical trials.
\end{abstract}

Data used in preparation of this article were obtained from the Alzheimer's Disease Neuroimaging Initiative database (adni.loni.usc. edu). As such, the investigators within the ADNI contributed to the design and implementation of ADNI and/or provided data but did not participate in analysis or writing of this report. A complete listing of ADNI investigators can be found at: http://adni.loni.usc.edu/wpcontent/uploads/how_to_apply/ADNI_Acknowledgement_List.pdf.

Supplementary information The online version of this article (https:// doi.org/10.1038/s41380-020-0818-1) contains supplementary material, which is available to authorized users.

Pedro Rosa-Neto

pedro.rosa@mcgill.ca

Extended author information available on the last page of the article

\section{Introduction}

Alzheimer's disease (AD) is defined by the presence of amyloid and tau pathologies that lead to neuronal injury or neurodegeneration and cognitive decline. Although the exact aetiology of $\mathrm{AD}$ is still being debated, the most widely accepted amyloid cascade hypothesis rests on amyloid-beta $(\mathrm{A} \beta)$ as the initiating event leading to a cascade of $\mathrm{AD}$ pathophysiological processes [1]. This hypothesis has gained strong support based on the multitude of biomarker-based evidence suggesting that $\mathrm{A} \beta$ abnormality occurs decades before the onset of AD [2]. This evidence suggests that the $\mathrm{A} \beta$ pathology correlates only weakly with neuronal injury or neurodegeneration but its downstream tau pathology is more strongly associated with neurodegeneration and consequently cognitive decline [3]. As such, the new NIA-AA research framework $\mathrm{A} / \mathrm{T} / \mathrm{(N)}$ focuses on measurable neuropathologic biomarkers to 
define and track patients along the $\mathrm{AD}$ continuum for staging purposes [4]. Following this framework, A (aggregated $\mathrm{A} \beta$ ) and $\mathrm{T}$ (aggregated tau) define $\mathrm{AD}$ and can be measured using positron emission tomography (PET) or cerebrospinal fluid (CSF) biomarkers, while $N$ (neuronal injury or neurodegeneration) is not specific to $\mathrm{AD}$ but provides staging of $\mathrm{AD}$ pathophysiological processes and can be measured using magnetic resonance imaging (MRI), $\left[{ }^{18} \mathrm{~F}\right]$ FDG-PET, and CSF total tau (T-tau) [4]. Effectively, the biomarkers of $N$ allow predicting cognitive decline or conversion for clinical trial enrichment [5].

Recent studies suggest neurofilament light chain (NFL) as a putative biomarker for neuronal injury or neurodegeneration. NFL is a subunit of the neurofilament that plays an important role in axonal and dendritic branching and growth, and axonal integrity [6,7]. Elevated NFL concentration in CSF has been associated not only with the axonal damage but also with neuronal injury, brain atrophy and disease severity in various neurological disorders, including $\mathrm{AD}$, and in their respective animal models [8-16]. Importantly, it has already been demonstrated that CSF and plasma NFL levels are correlated to each other and are elevated in AD [17].

To maximize the utility of a novel biomarker and its proper use in $\mathrm{AD}$ research and therapeutic trials, it is imperative to compare it with other well-established biomarkers. In the case of NFL, this would facilitate its incorporation among the existing biomarkers [4]. Current evidence on the influence of $\mathrm{A} \beta$ pathology on NFL levels has been conflicting. Some previous studies suggest that NFL levels may be independent of $A \beta$ pathology as there was no statistical difference between $A \beta$ - and $A \beta+$ subjects in the clinical spectrum of sporadic $\mathrm{AD}[11,18]$. However, a recent study showed that CSF and serum NFL levels were increased in pre-symptomatic familial $\mathrm{AD}$ mutation carriers and the rate of increase in the serum NFL was able to discriminate the mutation carriers from non-carriers almost a decade before the expected onset of symptoms [19]. Furthermore, a recent study on the Alzheimer's Disease Neuroimaging Initiative (ADNI) cohort showed that longitudinal increases in plasma NFL levels correlate with baseline CSF indices of brain amyloidosis [20]. Moreover, it was revealed that NFL concentration was negatively associated with grey matter volumes of a priori defined regions of interest (ROI) that are vulnerable in $\mathrm{AD}$-hippocampus, precuneus, or temporal composite regions (entorhinal, inferior temporal, middle temporal, and fusiform cortex) [11, 17, 19]. However, the empirical evidence on the NFL levels association with reduced grey matter density at a voxel-based regional level in the context of $\mathrm{A} \beta$ pathology is largely missing. Although NFL elevation is an indicator of the presence of neurodegenerative processes, this fluid biomarker lacks the topographical information present in $\left[{ }^{18} \mathrm{~F}\right] \mathrm{FDG}$-PET or MRI scans [21, 22]. This is an important limitation since plasma or CSF NFL levels might reflect disease-specific grey or white matter neurodegenerative processes or diseaseindependent healthy aging process [22-24]. Understanding the topographical correlates between NFL and neuronal injury or neurodegeneration in the context of $\mathrm{A} \beta$ pathology is crucial to interpret NFL levels as a novel fluid biomarker for neuronal injury or neurodegeneration in $\mathrm{AD}$ research and therapeutic trials.

In this study, we elucidate the contributing role of $A \beta$ pathology on neuronal injury reflected by NFL concentration and grey matter density maps. In addition, we illustrate voxel-based correlates between NFL and grey matter density maps in humans and McGill-Thy1-APP transgenic (Tg) rat model [25]. This Tg model is ideal to study the effect $A \beta$ alone as it progressively develops $A \beta$ pathology comparable to $\mathrm{AD} A \beta$ pathology without the presence of tau pathology or minimal cell death [25-27]. The present study highlights that NFL concentration reflects neuronal injury processes in the regions that are vulnerable to $\mathrm{AD}$ in the presence of $\mathrm{A} \beta$ pathology.

\section{Materials and methods}

\section{Subjects}

\section{Human dataset}

Data used in the preparation of this article were obtained from the ADNI (adni.loni.usc.edu). The ADNI was launched in 2003 by the National Institute on Aging (NIA), the National Institute of Biomedical Imaging and Bioengineering (NIBIB), the Food and Drug Administration (FDA), private pharmaceutical companies and non-profit organizations, as a $\$ 60$ million, 5-year public-private partnership. ADNI is the result of efforts of many co-investigators from a broad range of academic institutions and private corporations, and subjects have been recruited from over 50 sites across the USA and Canada. For up-to-date information, see www.adni-info.org.

The criteria for cognitively normal $(\mathrm{CN})$ classification are mini-mental state examination (MMSE) scores between 24 and 30 (inclusive) without cognitive complaints or observable anomalies. The criteria for the diagnosis of mild cognitive impairment (MCI) included the presence of memory complaints, MMSE scores between 24 and 30 (inclusive), objective memory loss, Clinical Dementia rating (CDR) of 0.5 , preserved activities of daily living, and absence of dementia. Mild AD dementia subjects had MMSE scores between 20 and 26 (inclusive), CDR of 0.5 or 1.0, and fulfilled the NINCDS/ADRDA criteria for probable AD. 


\section{Animal dataset}

All procedures described here were performed in compliance with ethics protocols approved by McGill Animal Care Ethics Committee and following the Canadian Council on Animal Care guidelines. All rats were housed at the Douglas Mental Health University Institute animal facility on a 12/12 h light/darkness cycle with ad libidum access to food and water. A starting sample of 26 animals was included in this study based on the effect size estimated from the previous study [12]. Eleven were wild type (WT) animals and 15 were homozygous McGill-R-Thy1-APP Tg rats were randomly selected. All the animals were on Wistar background for the study. All the animal procedures were completed blinded to the genotype.

\section{Inclusion and exclusion criteria}

\section{Human}

ADNI inclusion and exclusion criteria can be found at (www.adni-info.org). For our study, we included $83 \mathrm{CN}$, $160 \mathrm{MCI}$, and $73 \mathrm{AD}$ individuals who have both CSF and plasma NFL, CSF $A \beta_{42}$, CSF phosphorylated tau (p-tau), and structural MRI data available. For plasma NFL analysis, we identified two $\mathrm{CN}$, eight $\mathrm{MCI}$, and four $\mathrm{AD}$ subjects as outliers (two standard deviations away from the mean) who were excluded in the analysis.

\section{Animals}

All animal data points that overlap with any sign of brain tumour development or had a major surgery to remove tumour were excluded within the longitudinal data points (four WT and seven Tg). Last, all animals that were identified as an outlier (two standard deviations away from the mean) were excluded (two WT).

\section{Imaging acquisition and processing}

\section{Human}

Details on the image acquisition parameters can be found here (http://adni.loni.usc.edu/methods). All T1-weighted MRIs were processed using an in-house processing pipeline and underwent non-uniformity correction [28], brain masking [29], normalization to the Montreal Neurological Institute (MNI) space using affine and nonlinear transformation with Advanced Normalization Tools (ANTs) registration tools [30, 31], and segmented using three-tissue priors in MNI space (grey matter, white matter, and CSF). Our in-house pipeline based on Medical Imaging NetCDF (MINC) toolkits (www.bic.mni.mcgill.ca/ServicesSoftware) generated Voxel-based morphometry (VBM) images representing grey matter density maps. In brief, a log Jacobian determinant was derived based on the nonlinear vector field from the previously mentioned pipeline. Then, it was transformed into a scalar, modulated with grey matter probability mask, and smoothed with $8 \mathrm{~mm}$ full-width at half-maximum (FWHM). Last, three ROIs from the MNI template atlas were used to replicate the previous findings in the literature: (1) temporal composite (Temp.Comp; bilateral middle and inferior temporal gyri, fusiform and entorhinal cortices, and hippocampi), (2) Entorhinal cortices, and (3) hippocampi [11, 17, 20].

\section{Animals}

All MRI images were acquired using Bruker 7T BioSpec $70 / 30$ USR dedicated for small animal. All rats were under $1.5 \%$ isoflurane anaesthesia during the scan after a $5 \%$ anaesthesia induction.

All structural images were obtained at 15 months using the Bruker standard 3D-True Fast Imaging with Steady State Precession pulse sequence (3D-TruFISP). A rootmean-square image of eight phases acquisition was performed. Each angle acquisition was acquired in a field-ofview of $36 \times 36 \times 36 \mathrm{~mm}$ with a matrix of $180 \times 180 \times 180 \times$ of TE/TR of $2.5 / 5.0 \mathrm{~ms}$ with a flip angle of $30^{\circ}$ and NEX of 2. The final image was an average of the 16 acquisitions with a final $250 \mu \mathrm{m}$ isotropic resolution and a total acquisition time of 46 min scan.

All images were processed using MINC toolkits (www. bic.mni.mcgill.ca/ServicesSoftware). All individual MRI images were aligned with rigid body transformation, affine, and nonlinear transformation, and matched to generate an average population-based template based on Pydpiper [32].

Nonlinear vector field was used to create a log Jacobian determinant for deformation-based morphometry (DBM) representing local volume difference in each subject [32]. Considering rodent brain has white matter in limited regions, these local changes in the neocortex were considered grey matter changes. Finally, $1 \mathrm{~mm}$ FWHM smoothing was applied. Then DBM was transformed to a scalar as VBM values for ROI analysis. Last, three ROIs based on the Paxino and Watson atlas 6th edition were used: (1) temporal composite (Temp.Comp; bilateral temporal associative cortices and hippocampi), (2) Entorhinal cortices, and (3) hippocampi.

\section{Fluid collection and analysis}

\section{Human CSF}

CSF collection, processing, and storage procedures have been described previously (www.adni-info.org). CSF A $\beta_{42}$ 
and CSF p-tau concentrations were measured using the Elecsys on a cobas e 601 analyzer as described previously $[33,34]$. CSF NFL concentration was measured using a commercially available enzyme-linked immunosorbent assay (NF-light; UmanDiagnostics) as described by the manufacturer. The measurements were performed by boardcertified laboratory technicians, who were masked to clinical data, using one batch of reagents. Intrabatch coefficients of variation were below $10 \%$. Amyloid positivity was classified based on the previously established CSF p-Tau/ $\mathrm{A} \beta_{42}$ ratio cutoff threshold (CSF p-Tau/A $\left.\beta_{42}>0.025\right)$ [34].

\section{Human plasma NFL}

Plasma NFL collection, processing, and storage procedures have been described previously (www.adni-info.org). In brief, plasma NFL concentration was measured using an inhouse single-molecule array (Simo) method, as described previously in detail [35]. The measurements were performed by board-certified laboratory technicians, who were masked to clinical data, using one batch of reagents. For runs in this study, the coefficient of variations (CV) for the low $(11.0 \mathrm{ng} / \mathrm{L})$ quality control (QC) sample was $6.2 \%$ for intra-assay $\mathrm{CV}$ and $9.0 \%$ for inter-assay $\mathrm{CV}$, while the corresponding values for the high $(173.0 \mathrm{ng} / \mathrm{L}) \mathrm{QC}$ sample were $4.9 \%$ for intra-assay CV and $7.2 \%$ for inter-assay CV.

\section{Animals CSF}

All animals were anesthetized with a 5\% isoflurane induction for 5-8 $\mathrm{min}$ and placed in a stereotaxic apparatus. The CSF samples (100-150 $\mu \mathrm{L})$ were collected via direct puncture through the cisterna magna at 10-18 months of age.

Rat CSF NFL concentration was determined using the inhouse Simoa NFL assay which has been described in detail previously [16], with some modifications. Briefly, samples were diluted 100x with assay diluent and incubated for 35 min with paramagnetic carboxylated beads (Quanterix Corp, Boston, MA, USA) coated with a mouse antineurofilament light antibody (UD1, UmanDiagnostics, Umeå, Sweden) and a biotinylated mouse antineurofilament light antibody (UD2, UmanDiagnostics) in a Simoa HD-1 instrument (Quanterix). The bead-conjugated immunocomplex was thoroughly washed before incubation with streptavidin-conjugated $\beta$-galactosidase (Quanterix). Thereafter, the bead complex was washed and resorufin $\beta$-D-galactopyranoside (Quanterix) was added. The immunocomplex was applied to a multi-well array designed to enable imaging of every single bead. The average number of enzymes per bead (AEB) of samples was interpolated using the calibrator curve constructed by AEB measurements on bovine NFL (UmanDiagnostics) serially diluted in assay diluent. Samples were analyzed 'blind' and in duplicate using one batch of reagents. The average repeatability coefficient of variation of a sample with a mean concentration of $18,102 \mathrm{pg} / \mathrm{mL}$ was $8.5 \%$ and intermediate precision was $8.8 \%$, and for a sample with a mean concentration of $9460 \mathrm{pg} / \mathrm{mL}$, repeatability was $8.1 \%$ and intermediate precision was $11.5 \%$. All samples analyzed were above the lower limit of quantification (LLoQ).

Rat CSF concentrations of $A \beta_{42}$ and $A \beta_{40}$ were measured using Quanterix kits according to kit instructions (Simoa $\mathrm{A} \beta_{42} 2.0211$ and Simoa $A \beta_{40} 2.0218$, Quanterix) with a CSF dilution of $100 \times$ using the provided assay diluents. For $\mathrm{A} \beta_{42}$ CSF measurements, the average repeatability coefficient of variation of a sample with a mean concentration of $1650 \mathrm{pg} / \mathrm{mL}$ was $3.2 \%$ and intermediate precision was $9.2 \%$, and for a sample with a mean concentration of $641 \mathrm{pg} / \mathrm{mL}$, repeatability was $10.9 \%$ and intermediate precision was $16.6 \%$. For $A \beta_{40}$ CSF measurements, repeatability for a sample with a concentration $5160 \mathrm{pg} / \mathrm{mL}$ was $17.2 \%$, and intermediate precision $17.2 \%$, and for a sample with a concentration of $3975 \mathrm{pg} / \mathrm{mL}$ repeatability and intermediate precision was $7.3 \%$ and $11.8 \%$, respectively. For both assays, a number of samples were below the assigned assay limit of quantification and assigned a concentration of half of the LLoQ.

\section{Statistical analysis}

Potential difference in the number of male and female in each group was tested based on a chi-square test for both humans and animals as well as APOE\&4 status in humans. We also tested whether there were differences in age, education, and MMSE scores for humans based on a two-sided ANOVA. We tested if WT and Tg had any difference in age using an unpaired two-sided $t$ test. We $\log$ transformed NFL in CSF and plasma, as well as CSF A $\beta_{42}$ and p-tau.

In animals, linear mixed effect models with a random intercept were performed to measure longitudinal changes in CSF A $\beta_{42 / 40}$ and CSF NFL log: (1) CSF A $\beta_{42 / 40} \sim$ Age + (1lanimals); (2) CSF NFL (log) Age (WT or Tg) + (1l animals); (3) CSF NFL (log) Age + Genotype + Age $\times$ Genotype + (1lanimals). The association between CSF NFL and CSF $A \beta_{42 / 40}$ was investigated based on a linear regression model. Weight was retained as a variable only in the model showing the association between CSF NFL and CSF $A \beta_{42 / 40}$ as it was shown to have a significant effect.

In animals and humans, voxel-based linear regression models were performed to detect the group contrast in DBM and VBM, respectively. Furthermore, a voxel-based linear regression was performed between DBM and CSF NFL log at 15 months for animals, and VBM and CSF or plasma NFL levels for humans: animal model: CSF NFL log 
DBM; human model: NFL (CSF or plasma) $\sim$ VBM + covariates in each group as well as combining all groups with adjusting for the diagnosis. Similarly, we used those same models as the voxel-based models to replicate the previous findings using the Temp.Comp, entorhinal, and hippocampus ROIs with FDR correction for animals and humans $[11,17,20]$. In addition, we applied bootstrapping technique in the group that showed significant association between NFL and VBM, running the standardized ROIbased models 2000 times to compare the effect size between $\mathrm{A} \beta-$ vs $\mathrm{A} \beta+$. All voxel-based and ROI-based analyses and linear regression models examining the grey matter density and NFL concentrations in humans were corrected for age, sex, APOE 4 status, education, CSF p-tau (log), and the difference in dates between the NFL measurements and MRI scan. Furthermore, additional CSF A $\beta_{42}$ :p-tau interaction term was tested to exclude the effect of $p$-tau and $\mathrm{A} \beta_{42}$ interaction when the term was significant.

All voxel-based analyses were conducted using VoxelStats and corrected for multiple comparison using random field theory with peak threshold at $p=0.05$ and cluster threshold at $p=0.05$ for rats and $p=0.005$ for human [36]. All the statistical analyses were conducted using R 3.4.4.

\section{Results}

\section{Demographic}

Table 1 summarizes the demographic of the participants included in the study. There was no difference in age or education between groups. However, the number of males and females was different between $\mathrm{CN} A \beta+$ vs MCI A $\beta-$ and MCI $A \beta-$ vs $A D A \beta+$. APOE 44 status was different between groups except for $\mathrm{CN} A \beta-$ vs MCI $\mathrm{A} \beta-, \mathrm{CN} A \beta+$ vs MCI $\mathrm{A} \beta+$, and MCI $\mathrm{A} \beta+$ vs $\mathrm{AD} A \beta+$. Furthermore, average MMSE score was not different between $\mathrm{CN} A \beta-$ vs $\mathrm{CN} A \beta+$ and MCI $A \beta-$ vs MCI $A \beta+$, whereas all other comparisons showed significant differences.

For the animal data, there was no difference in age, sex, or weight (Table 1).

\section{Longitudinal CSF biomarker changes and their association and grey matter changes in animals}

In McGill-Thy1-APP $\mathrm{Tg}$ rats only, a linear mixed effect analysis showed a significant longitudinal decline in CSF $\mathrm{A} \beta_{42 / 40}$ from 10 months to 18 months $(\beta=-0.01$, standard error (s.e) $=0.0045, t(63)=-2.09, p=0.041$ ) (Fig. 1a). On the other hand, the longitudinal CSF NFL concentration significantly increased in both WT $(\beta=+0.05$, s.e $=0.012$, $t(27)=4.06, p=0.0004)$ and $\operatorname{Tg}(\beta=+0.04$, s.e $=0.006$, $t(64)=5.63, p<0.0001)($ Fig. 1b). When we analyzed both

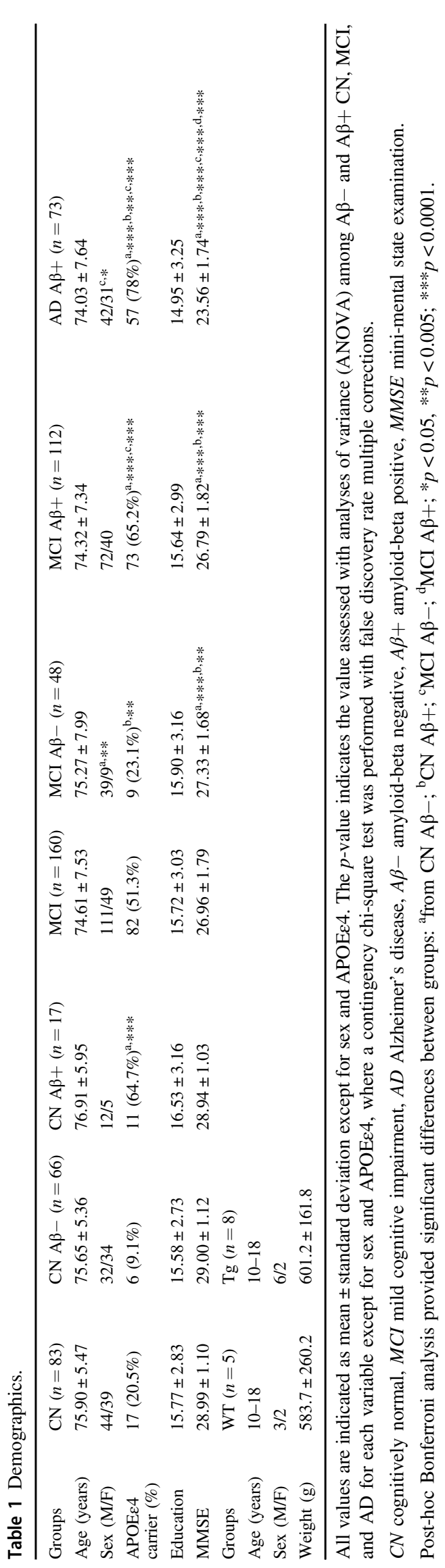



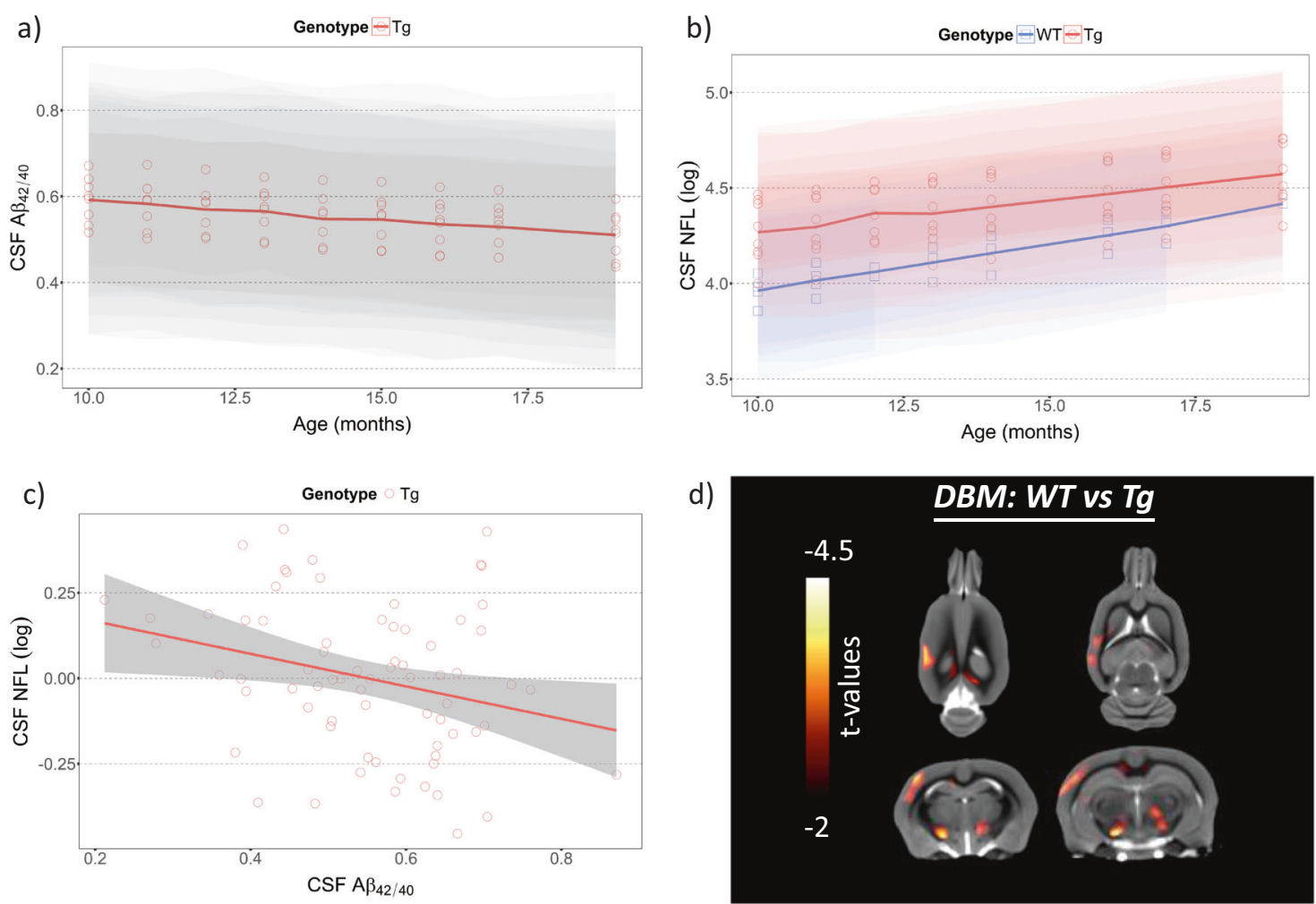

d)

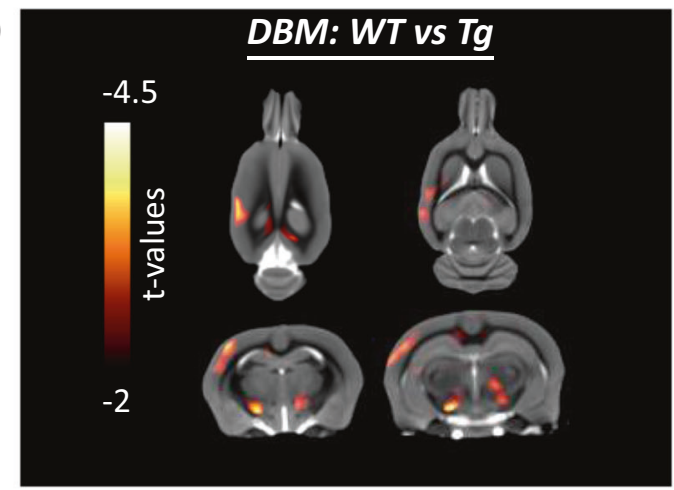

Fig. 1 Natural progression in CSF biomarkers and grey matter density changes in control and Tg animals. a, b Longitudinal analyses on CSF $A \beta_{42 / 40}$ and NFL concentrations, $\mathbf{c}$ an association between CSF $A \beta_{42 / 40}$ and NFL concentration, and $\mathbf{d}$ voxel-based group

contrast between WT and Tg in DBM with the $t$-statistic scale. The shades in the regression lines represent $95 \%$ confidence intervals of the model.

groups into one model by including an interaction term between the group and time, the CSF NFL concentration was progressively increased over time $(\beta=+0.05$, s.e $=$ $0.01, t(93) 4.38, p<0.0001)$ while $\mathrm{Tg}$ had a significantly greater CSF NFL concentration compared to WT $(\beta=$ +0.43 , s.e $=0.2, t(90)=2.14, p=0.035$ ) (Fig. 1b). However, the interaction between the genotype effect and longitudinal change in CSF NFL concentration was not significant. Similar to previous human findings, the significant inverse association between CSF NFL and CSF $\mathrm{A} \beta_{42 / 40}$ was observed when adjusted for weight $(\beta=-0.5$, s.e $=0.2, t(68)=-2.40, p=0.02)$ (Fig. 1c).

Voxel-based group contrast in DBM at 15 months showed significant reduction in parietotemporal cortex $(t$ $(11)=3.2, p=0.008)$, bilateral dorsal hippocampi $(t(11)=$ 2.3, $p=0.042$ ), and striatum in Tg compared to WT with the greatest contrast seen in thalamus $(t(11)=5, p=$ 0.0004) (Fig. 1d and Supplementary Fig. 1 and Table 1).

\section{CSF NFL and DBM association is modulated by amyloid in AD-vulnerable regions in $\mathrm{Tg}$}

Increased CSF NFL concentration was significantly associated with reduced DBM only in Tg, encompassing the parietotemporal cortex, hippocampus, striatum, thalamus,

and cerebellum in the voxel-based analysis. The greatest effect was observed in the parietotemporal cortex $(t(6)=13$, $p=0.0001)$ and hippocampus $(t(6)=8, \quad p=0.0002)$ (Fig. 2a and Supplementary Fig. 2 and Table 2). The ROIbased linear regression analyses revealed significant negative associations between CSF NFL concentration and the Temp.Comp VBM $(\beta=-4.09$, s.e $=1.29, t(6)=$ $-3.18, p=0.037)$, entorhinal cortex $(\beta=-3.50$, s.e $=$ $1.17, t(6)=-2.99, p=0.037)$, and hippocampus VBM $(\beta=-3.90$, s.e $=1.58, t(6)=-2.47, p=0.048)$ only in $\mathrm{Tg}$ while WT showed no association. (Fig. 2b).

\section{VBM is reduced in AD-vulnerable regions in humans}

A voxel-based group contrast on VBM between $\mathrm{CN}$ and MCI revealed a significant reduction in the hippocampus, precuneus/posterior cingulate cortex (PCC), medial frontal cortex, and lateral temporal cortex (Fig. 3a and Supplementary Fig. 3 and Table 3). The greater difference was seen in $\mathrm{CN}$ and $\mathrm{AD}$ contrast where the clusters extended to the orbital frontal cortex, basal lateral temporal cortex (including entorhinal cortex), as well as the parahippocampal gyrus (Fig. 3b and Supplementary Fig. 4 and Table 4). When we compared MCI and $\mathrm{AD}$, the latter showed a significant VBM reduction in precuneus/PCC, 


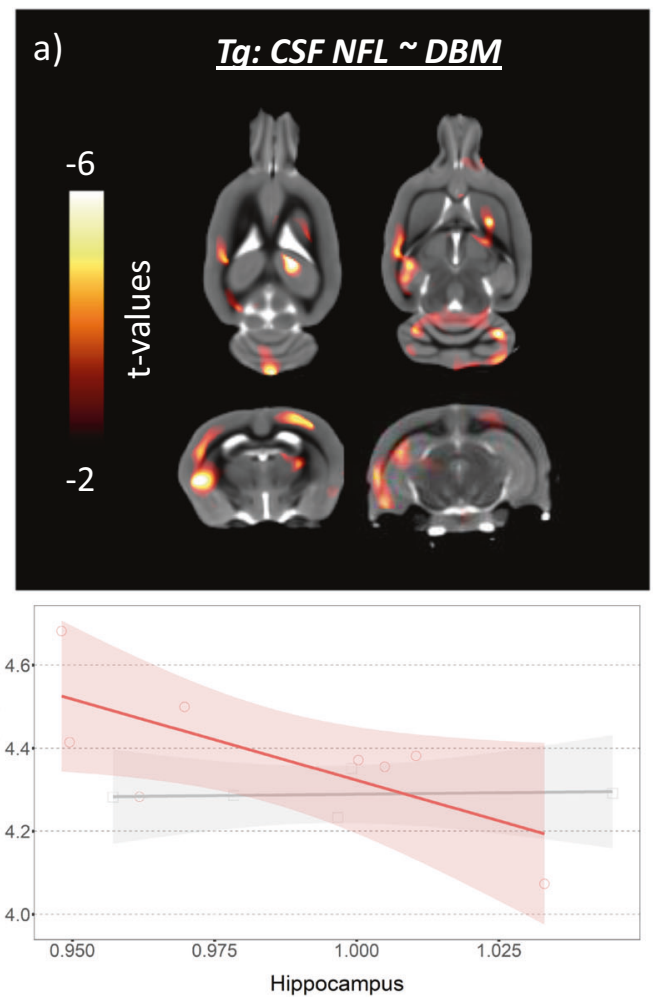

Fig. 2 Increased CSF NFL concentrations are associated with reduced grey matter density in transgenic animals. a The association between CSF NFL concentration and DBM at 15 months of age in Tg with the $t$-statistic scale. b The association between CSF b)
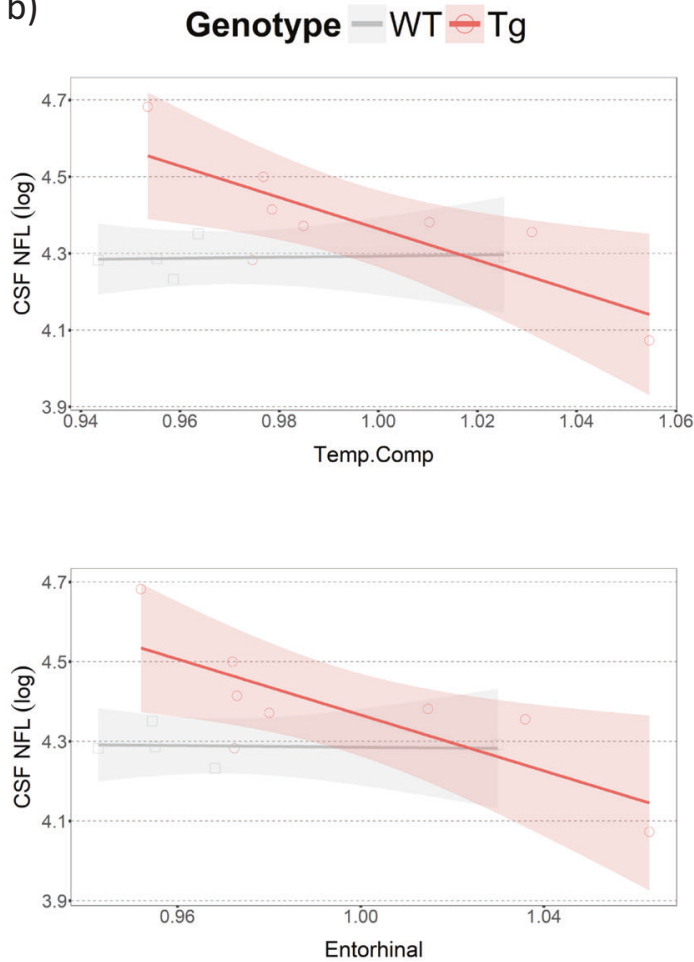

NFL concentration and ROIs from Temp.Comp, entorhinal, and hippocampus VBM in WT and Tg. The shades in the regression lines represent $95 \%$ confidence intervals of the model.

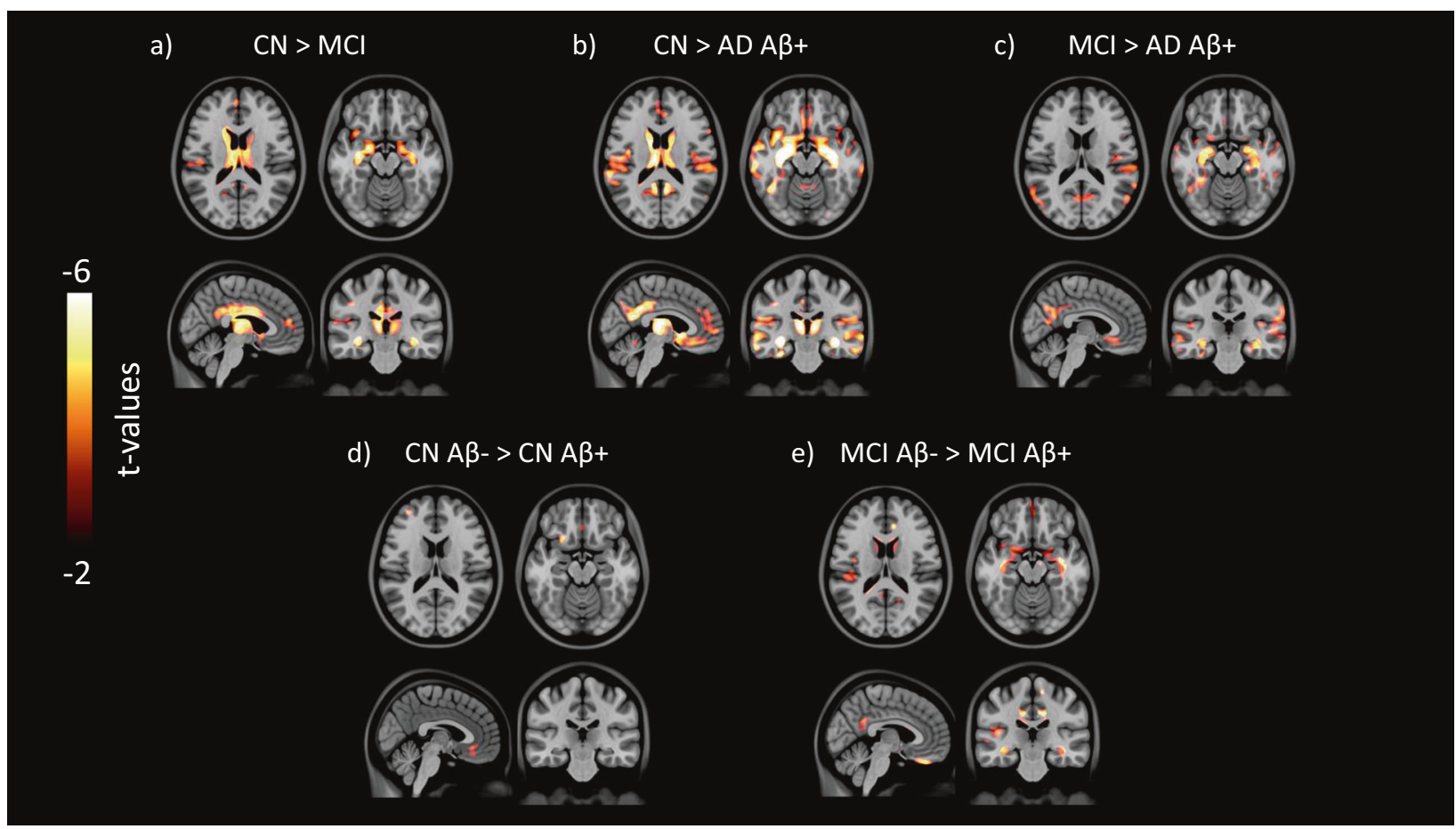

Fig. 3 Grey matter density changes in AD spectrum. Significant group contrast maps showing the difference in the VBM between a CN and $\mathrm{MCI}, \mathbf{b} \mathrm{CN}$ and $\mathrm{AD} \mathrm{A} \beta+, \mathbf{c} \mathrm{MCI}$ and $\mathrm{AD} \mathrm{A} \beta+$, and $\mathbf{d} \mathrm{CN} \mathrm{A} \beta-$ and $\mathrm{A} \beta+\mathbf{e} \mathrm{MCI} \mathrm{A} \beta-$ and MCI $\mathrm{A} \beta+$. All of the result images are represented on the same $t$-statistic scale. 
a)

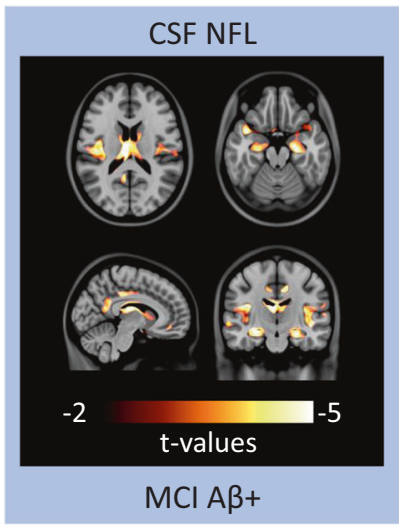

Diagnostic Group $=M C I A \beta-=M C I A \beta+$

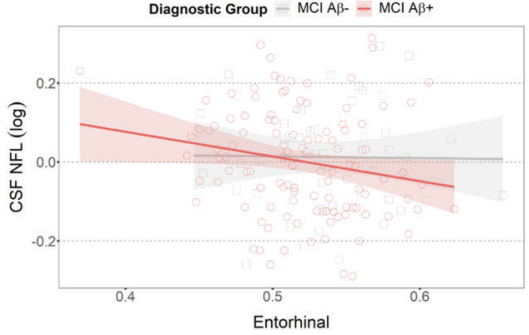

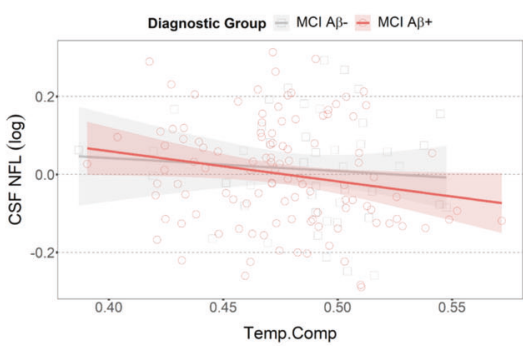

b)
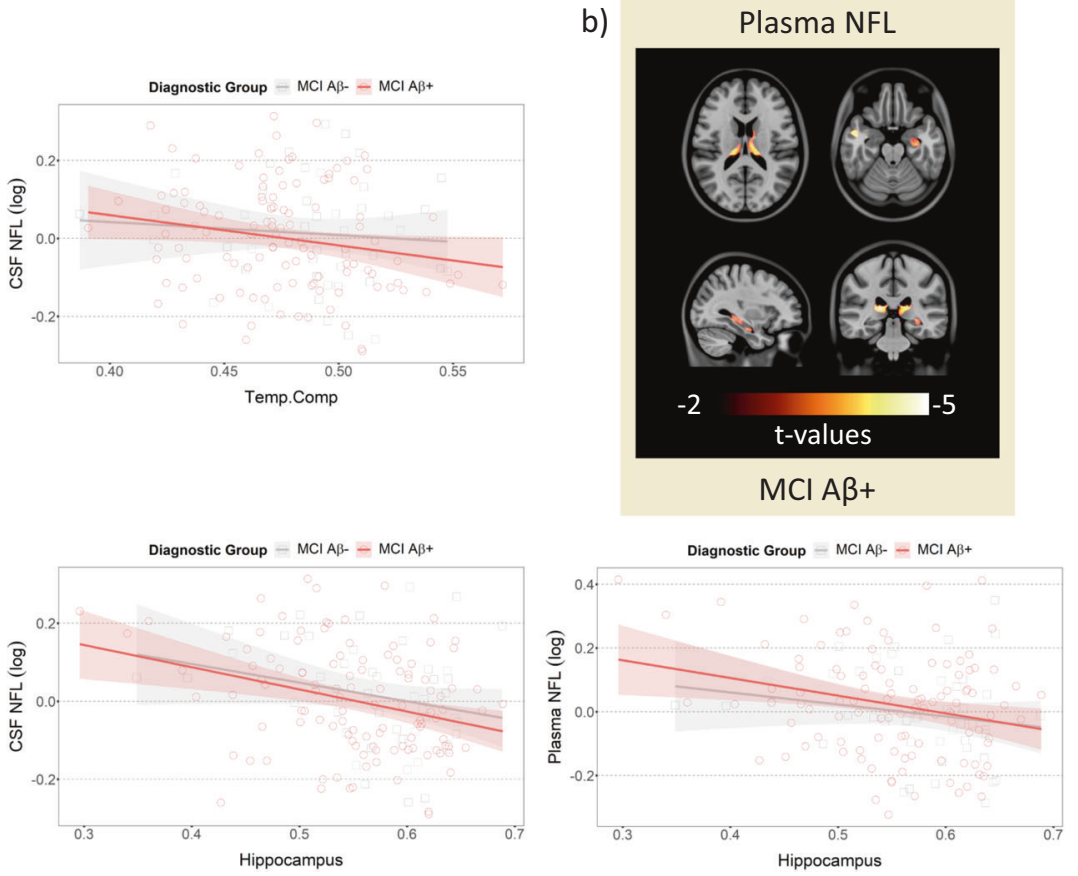

Fig. 4 Increased NFL concentrations are associated with reduced grey matter density in MCI A $\mathbf{\beta}+$. a Voxel-based maps showing significant associations between CSF NFL concentration and VBM in MCI A $\beta+$ and ROI-based analyses in MCI A $\beta$ - (in grey) and MCI A $\beta$ + (in red) showing the association between CSF NFL and Temp.
Comp, Entorhinal cortex, or hippocampus. b Voxel-based maps showing significant associations between plasma NFL concentration and VBM in MCI A $\beta+$ and ROI-based analyses in MCI A $\beta$ - (in grey) and $\mathrm{MCI} A \beta+$ (in red) showing the association between plasma NFL and hippocampus. a)

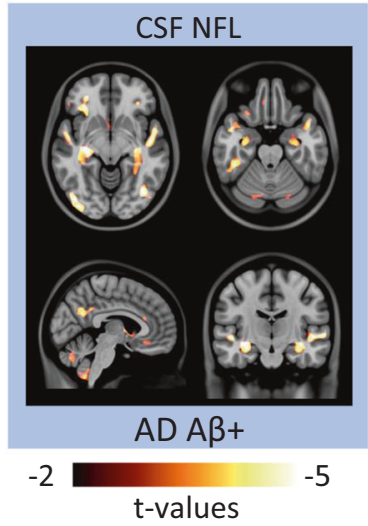

b)

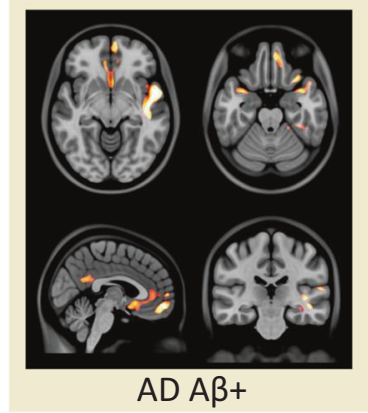

c)
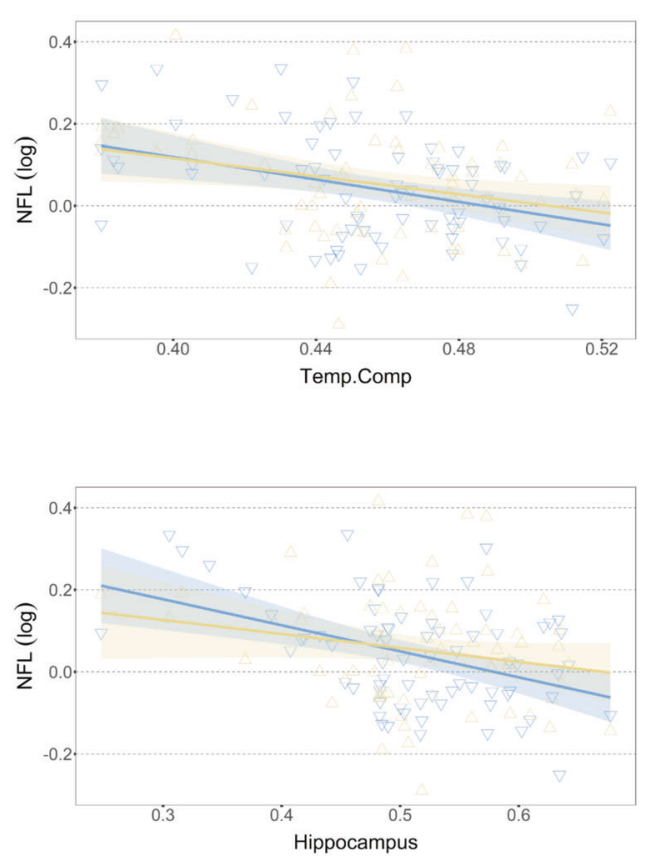

d)

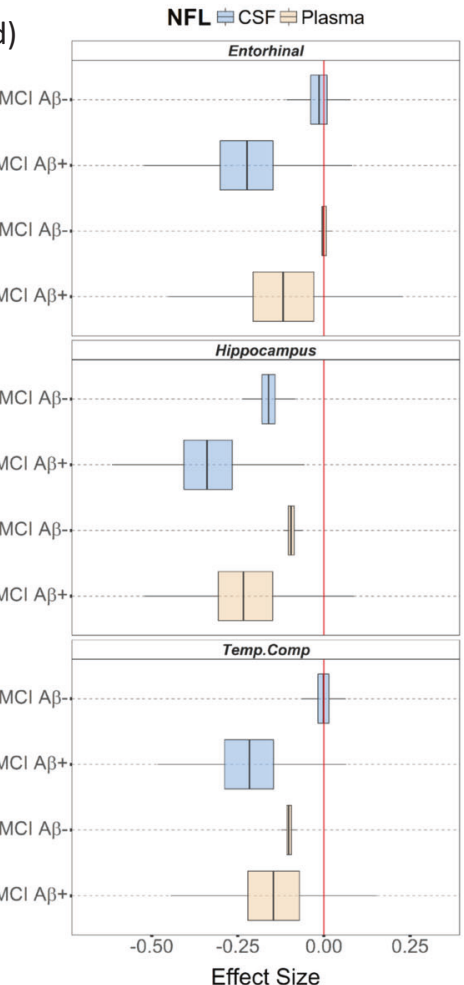

Fig. 5 Increased NFL concentrations are associated with reduced grey matter density in AD A $\beta+$. Voxel-based maps showing significant associations between a CSF NFL concentration and VBM, b plasma NFL concentration and VBM, and $\mathbf{c}$ the association between CSF NFL (blue) or plasma NFL (yellow) and Temp.Comp or hippocampus ROIs in $\mathrm{AD} A \beta+$. The shades represent a 95\% confidence interval of the regression models. $\mathbf{d}$ Bootstrap analysis showing the difference in the effect sizes between MCI $A \beta+$ and $A \beta-$ in CSF or plasma NFL association with VBM. 
medial frontal cortex, basal lateral temporal cortex, inferior parietal cortex, and hippocampus (Fig. 3c and Supplementary Fig. 5 and Table 5). Within the same clinical diagnostic group, $\mathrm{CN}$ and $\mathrm{MCI}$ showed a difference where $\mathrm{CN} \mathrm{A} \beta+$ had a greater VBM reduction in the medial frontal cortex and MCI $\mathrm{A} \beta+$ had greater VBM reduction in the medial frontal cortex, lateral temporal cortex, and right hippocampus compared to the counter $A \beta$ - group, respectively (Fig. 3d, e and Supplementary Figs. 6 and 7 and Tables 6 and 7).

\section{The NFL concentration and VBM association is modulated by amyloid in AD-vulnerable regions in humans}

In MCI A $\beta+$ cases, increased CSF NFL concentration was significantly associated with reduced VBM in the orbitofrontal cortex, lateral temporal cortex, precuneus/PCC, hippocampus and thalamus based on the voxel-based analysis (Fig. 4a and Supplementary Fig. 8 and Table 8). Also, increased plasma NFL concentration was significantly associated with reduced VBM in the lateral temporal cortex and right hippocampus $(t(99)=-3.3, p=0.0034)$ in MCI A $\beta+$ (Fig. 4b and Supplementary Fig. 9 and Table 9). The ROI-based linear regression analyses revealed significant negative associations between CSF NFL concentration and the Temp.Comp VBM $(\beta=-0.93$, s.e $=0.39, t(104)=$ $-2.38, p=0.018)$, entorhinal cortex $\operatorname{VBM}(\beta=-0.85$, s.e $=0.32, t(104)=-2.51, p=0.015)$, and both CSF and Plasma NFL were associated with hippocampus VBM $(\beta=$ -0.90 , s.e $=0.20, t(104)=-4.59, p<0.0001 ; \beta=-0.70$, s.e $=0.24, t(99)=-3.03, p<0.013$, respectively) in MCI $\mathrm{A} \beta+$ (Fig. $4 \mathrm{a}, \mathrm{b})$. In the MCI $\mathrm{A} \beta+$, only the plasma NFL and ROI-VBM analyses showed a significant CSF $A \beta_{42}$ :ptau interaction. Even when this term was included the results stayed the same.

In $\mathrm{AD} \mathrm{A} \beta+$, both CSF and plasma NFL levels were significantly associated with VBM results in dorsal lateral frontal cortex, lateral temporal cortex, precuneus/PCC, medial frontal cortex, angular gyrus, hippocampus, and cerebellar grey in the voxel-based analyses (Fig. 5a, b and Supplementary Figs. 10 and 11 and Tables 10 and 11). Moreover, CSF NFL levels were significantly associated with occipital gyrus VBM measurements in AD $\mathrm{A} \beta+$ (Fig. 5a). The ROI-based linear regression analyses revealed significant negative associations between CSF and the Temp.Comp VBM $(\beta=-1.15$, s.e $=0.44, t(65)=$ $-2.69, p=0.016)$ and hippocampus $\operatorname{VBM}(\beta=-0.82$, s.e $=0.20, t(65)=-3.99, p<0.0001)$ while plasma NFL and VBM in the Temp.Comp and hippocampus ROIs showed a small trend $(t(61)=-1.88, t(61)=-1.81$, respectively, $p=0.15$ ).
Although $\mathrm{CN} A \beta-$ and $\mathrm{A} \beta+$ and $\mathrm{MCI} \mathrm{A} \beta-$ groups showed a significant association between NFL and VBM, the RFT-survived clusters were small. $\mathrm{CN} A \beta$ - showed a negative association between CSF NFL and lateral frontorbital cortex while $\mathrm{CN} A \beta+$ showed in the cingulate cortex (Supplementary Figs. 12 and 13 and Tables 12 and 13). MCI $A \beta$ - group showed a negative association between plasma NFL and VBM in the insular cortex only (Supplementary Fig. 14 and Table 14). When combining all the groups and adjusting for the diagnosis, only the $\mathrm{A} \beta+$ group showed a negative association between CSF or plasma NFL and precuneus/PCC, basolateral temporal cortex, medial frontal cortex, and striatum (Supplementary Figs. 15 and 16 and Tables 15 and 16).

\section{Greater effect sizes in $A \beta+$ groups and CSF NFL}

To compare the effect size of the association between CSF or plasma NFL and VBM in A $\beta$ - and $\mathrm{A} \beta+$, we applied bootstrapping to run the standardized ROI-based models 2000 times while matching the number of subjects between MCI $\mathrm{A} \beta-$ and $\mathrm{A} \beta+$. We selected the MCI group to compare because MCI $A \beta+$ showed significant results from the ROI analyses. In all three ROIs, MCI A $\beta+$ showed greater effect size in both CSF and plasma NFL and VBM associations compared to MCI A $\beta$ - (Fig. 5d). This corresponds with our animal results where only the Tg group showed significant associations between CSF NFL and VBM in the homologous ROIs (Fig. 2b). In addition, we applied bootstrapping to run standardized ROI-based models 2000 times in MCI $\mathrm{A} \beta+$ and $\mathrm{AD} A \beta+$. This revealed the association between CSF NFL and VBM had greater effect sizes in most of the regions compared to the association between plasma NFL and VBM (Supplementary Fig. 17).

\section{Discussion}

In this present study, we found an unbiased voxel-based association between NFL concentrations and grey matter density maps in $\mathrm{A} \beta$ pathology in humans and animals. NFL has been reported to be a novel neuronal injury or neurodegeneration biomarker but previous studies have only focused on the association between NFL concentrations and a priori ROIs volume over regions typically affected by $\mathrm{AD}$ $[11,17,19]$. Assessing the association between NFL concentrations and grey matter changes over the brain allowed us to probe whether the levels of NFL are driven by ADvulnerable regional neuronal injury or age-related neurodegeneration. Based on our empirical evidence from human and $\mathrm{Tg}$ animal model analyses, the $\mathrm{A} \beta$ was sufficient to elevate NFL levels and reduce grey matter density and they 
were associated in $\mathrm{AD}$-vulnerable regions in the presence of A $\beta$ pathology.

Considering that the level of NFL is influenced by other pathophysiological processes (i.e., tau pathology and neuroinflammation) that also are invariably present in clinically manifested $\mathrm{AD}$, it is difficult to fully dissect the degree of contribution from $\mathrm{A} \beta$ alone on NFL concentration $[8,10,18,37]$. Therefore, in this study, we investigated Tg rats with the McGill-R-Thy1-APP model in parallel with the human analyses, using the same methodological approach. Previously, this rat model was shown to have minimal cell death at 18 months of age, reduction in hippocampal volume, and loss of resting-state cingulate connectivity at 16-19 months of age in the presence of A $\beta$ pathology but without tau pathology [25-27]. This most likely reflects the synaptic dysfunction, neuronal injury, and network dysfunction caused by $\mathrm{A} \beta$ rather than cell death [26, 27, 38]. Here, our results revealed significant DBM results in the neocortex at 15 months of age and increased CSF NFL concentration in $\mathrm{Tg}$ as compared to WT. Therefore, this study strongly supports the notion that the $\mathrm{A} \beta$ pathology alone is sufficient to induce neuronal injury with minimal cell death. Association between the A $\beta$-induced CSF NFL concentrations increase and the DBM measurements reduction was evident in the neocortex and hippocampus where the $\mathrm{A} \beta$ pathology is prominent in this animal model. This strongly supports our findings that NFL reflects neuronal injury in $\mathrm{AD}$-related regions in the presence of $\mathrm{A} \beta$ pathology.

In addition, our novel findings from the first longitudinal animal CSF analyses revealed that the CSF NFL concentration significantly increased during the healthy aging process as indicated by the results in WT. Also, the CSF $\mathrm{A} \beta_{42 / 40}$ significantly decreased while CSF NFL concentration was significantly increased in Tg animals, showing the inverse relationship between the two biomarkers, thereby replicating the human finding [11]. Considering that the McGill-R-Thy1-APP Tg rat model displays only $\mathrm{A} \beta$ pathology without tau pathology, we demonstrated a modest but significant $A \beta$ effect on CSF NFL concentration in Tg compared to WT without the potential effect of tau pathology. Importantly, there was no significant interaction effect between genotype and age. This Tg model has been reported to start accumulating $\mathrm{A} \beta$ plaques between the age of 6 and 9 months; the soluble forms of $A \beta$ oligomers dominate prior to this point $[25,26,39]$. Here, we showed that CSF NFL concentration was already elevated before 10 months and remained high throughout the time over, which we collected our samples. This most likely represents the neurotoxic events induced by soluble $\mathrm{A} \beta$ oligomers, which in turn increase CSF NFL concentration during the early stage of the pathology in this animal model [38]. Therefore, our study suggests that the association between
$\mathrm{A} \beta$ and CSF NFL level is established at an early stage of the pathology. Interestingly, it has been reported that there is a significant interaction between the age and genotype on CSF NFL concentration in an APPPS1 mice model [12]. The discrepancy with our results could be due to much more aggressive A $\beta$ pathology accumulation in the APPPS 1 mice model as compared to the McGill-R-Thy1-APP rat model, in which the $\mathrm{A} \beta$ pathology develops at an equivalent rate as seen in the humans in McGill-R-Thy1-APP rat model [26]. Our longitudinal findings are in agreement with the other recently published longitudinal NFL measurements findings indicating that the rate of serum NFL change increases during the pre-clinical phase of the disease but plateaus before symptoms onset in familial AD, and that CSF NFL levels over time follow a U-shaped curve in sporadic AD $[19,40]$. Nonetheless, the animal models of $\mathrm{A} \beta$ pathology concurringly argue that $A \beta$ pathology leads to the increased CSF NFL concentration.

Neuronal injury or neurodegeneration is not an AD specific process. Besides AD, increased levels of NFL are also reported in other neurodegenerative conditions such as frontotemporal dementia, progressive supranuclear palsy, and Creutzfeldt-Jakob disease [9, 10, 13, 41, 42]. However, an increasing number of large-scale network analyses have shown a disease-specific vulnerability in an ensemble of regions for each of them $[22,23]$. Thus, it is imperative to study if an increased level of a fluid biomarker reflecting neuronal injury or neurodegeneration is linked to anomalies within the network affected by a given disease. Recent evidence based on the association between NFL levels and VBM measurements was in agreement with other studies using a priori defined ROIs in frontotemporal lobar degeneration and Huntington disease, showing that increased NFL concentration was associated with reduced frontal cortex and striatum VBM measurements, respectively. This supports the hypothesis that indeed increased NFL concentrations in those diseases follow neuronal injury in their specific network [10, 13]. To the best of our knowledge, our study is the first to describe voxel-based associations between the NFL levels and VBM measurements in regions targeted specifically by $\mathrm{AD}$. We show that the elevated NFL concentration is associated with reduced VBM measurements in the presence of $\mathrm{A} \beta$ pathology in the precuneus/PCC, medial frontal cortex, lateral temporal cortex, inferior parietal cortex, and hippocampus, which are all considered to be AD-vulnerable regions. It is important to note that these cortical regions are also form the default mode network on which AD pathological, functional, and structural abnormalities converge, leading to network failure [21, 22, 43-45]. We dichotomized $A \beta$ pathology based on the previously used cutoff points and included covariates, such as CSF p-tau and/or CSF $\mathrm{A} \beta_{42}$ :p-Tau interaction that could potentially confound the association between 
NFL levels and grey matter density maps, and yet, we found correlations only in the $\mathrm{A} \beta$ positive groups and in the ADvulnerable regions [34]. The reason why these regions are vulnerable in AD still remains poorly understood.

There are some important limitations to this study. Due to the modest effect size in the association between NFL concentration and grey matter density maps, the available number of individuals who have both CSF and plasma NFL concentration and MRI data in the ADNI database is overall low, which limited the statistical power of our study. Perhaps this weakness could explain why we observed a minimal association in $\mathrm{CN} \mathrm{A} \beta+$. Future studies with a greater number of participants and longitudinal data would be of interest. Another possible reason for the minimal association in $\mathrm{CN} A \beta+$ may be because some $\mathrm{CN}$ elderly individuals with high $A \beta$ pathology might actually have minimal levels of toxic soluble A $\beta$ oligomers [46]. Indeed, our animal results suggest that the increase in CSF NFL concentration occurs most likely due to the presence of soluble $A \beta$ oligomers as opposed to that of plaques $[38,39]$. In addition, we corrected the analysis for CSF p-tau levels and/or CSF A $\beta$ and p-tau interaction to exclude a potential effect of tau pathology on the association between NFL levels and grey matter density maps. However, it would be interesting to include tau-PET imaging data in a future study as it would allow to control for the different tau loads in different brain regions. Regarding our animal data, although the number of animals in WT and Tg cohorts was not significantly different initially, some of the WT animals developed pituitary tumours during the course of the study. An exploratory analysis revealed that this condition to be a confounding factor due to the significantly increased CSF NFL concentration in the animals that developed those pituitary tumours as compared to the healthy animals (data not included). Therefore, they were excluded in the group comparison analysis.

In summary, our evidence supports that elevated NFL levels in $\mathrm{AD}$ results from $\mathrm{A} \beta$-induced neuronal injury in AD-vulnerable regions. NFL is a sensitive biomarker capable of detecting the modest levels of neuronal injury linked to the healthy aging process or to pure $A \beta$ pathology. As such, our study supports the hypothesis that NFL is a novel biomarker of neuronal injury and neurodegeneration that may be utilized within the $\mathrm{A} / \mathrm{T} / \mathrm{N}$ classification scheme and for the evaluation of therapeutic efficacy in clinical trials.

Acknowledgements The authors would like to send a gratitude to EveMarie Charbonneau and her animal facility team for her technical support with animal care. This work was supported by the Canadian Institutes of Health Research (CIHR; FRN, 152985, PR-N), the Alzheimer's Association (NIRP-12-259245, PR-N), Fonds de Recherche du Québec-Santé (FRQS; Chercheur Boursier, PR-N). ACC acknowledges support from the Canadian Institutes for Health Research (CIHR grant-2016PJT-364544). KB holds the Torsten
Söderberg Professorship in Medicine at the Royal Swedish Academy of Sciences, and is supported by the Swedish Research Council (\#2017-00915), the Swedish Alzheimer Foundation (\#AF-742881), Hjärnfonden, Sweden (\#FO2017-0243), and a grant (\#ALFGBG715986) from the Swedish state under the agreement between the Swedish government and the County Councils, the ALF-agreement. $\mathrm{HZ}$ is a Wallenberg Academy Fellow supported by grants from the Swedish Research Council (\#2018-02532), the European Research Council (\#681712), Swedish State Support for Clinical Research (\#ALFGBG-720931) and the UK Dementia Research Institute at UCL. Data collection and sharing for this project was funded by the ADNI (National Institutes of Health Grant U01 AG024904) and DOD ADNI (Department of Defense award number W81XWH-12-2-0012). ADNI is funded by the National Institute on Aging, the National Institute of Biomedical Imaging and Bioengineering, and through generous contributions from the following: AbbVie, Alzheimer's Association; Alzheimer's Drug Discovery Foundation; Araclon Biotech; BioClinica, Inc.; Biogen; Bristol-Myers Squibb Company; CereSpir, Inc.; Cogstate; Eisai Inc.; Elan Pharmaceuticals, Inc.; Eli Lilly and Company; EuroImmun; F. Hoffmann-La Roche Ltd and its affiliated company Genentech, Inc.; Fujirebio; GE Healthcare; IXICO Ltd.; Janssen Alzheimer Immunotherapy Research \& Development, LLC.; Johnson \& Johnson Pharmaceutical Research \& Development LLC.; Lumosity; Lundbeck; Merck \& Co., Inc.; Meso Scale Diagnostics, LLC.; NeuroRx Research; Neurotrack Technologies; Novartis Pharmaceuticals Corporation; Pfizer Inc.; Piramal Imaging; Servier; Takeda Pharmaceutical Company; and Transition Therapeutics. The Canadian Institutes of Health Research is providing funds to support ADNI clinical sites in Canada. Private sector contributions are facilitated by the Foundation for the National Institutes of Health (www.fnih.org). The grantee organization is the Northern California Institute for Research and Education, and the study is coordinated by the Alzheimer's Therapeutic Research Institute at the University of Southern California. ADNI data are disseminated by the Laboratory for Neuro Imaging at the University of Southern California.

\section{Compliance with ethical standards}

Conflict of interest $\mathrm{KB}$ has served as a consultant or at advisory boards for Alector, Biogen, CogRx, Lilly, MagQu, Novartis and Roche Diagnostics, and is a co-founder of Brain Biomarker Solutions in Gothenburg $\mathrm{AB}$, a GU Venture-based platform company at the University of Gothenburg. HZ has served at scientific advisory boards of Roche Diagnostics, Wave, Samumed and $\operatorname{CogRx}$, has given lectures in symposia sponsored by Biogen and Alzecure, and is a co-founder of Brain Biomarker Solutions in Gothenburg AB, a GU Ventures-based platform company at the University of Gothenburg.

Publisher's note Springer Nature remains neutral with regard to jurisdictional claims in published maps and institutional affiliations.

Open Access This article is licensed under a Creative Commons Attribution 4.0 International License, which permits use, sharing, adaptation, distribution and reproduction in any medium or format, as long as you give appropriate credit to the original author(s) and the source, provide a link to the Creative Commons license, and indicate if changes were made. The images or other third party material in this article are included in the article's Creative Commons license, unless indicated otherwise in a credit line to the material. If material is not included in the article's Creative Commons license and your intended use is not permitted by statutory regulation or exceeds the permitted use, you will need to obtain permission directly from the copyright holder. To view a copy of this license, visit http://creativecommons. org/licenses/by/4.0/. 


\section{References}

1. Hardy J, Selkoe DJ. The amyloid hypothesis of Alzheimer's disease; progress and problems on the road to therapeutics. Science. 2002;297:353-6.

2. Jack CR, Knopman DS, Jagust WJ, Petersen RC, Weiner MW, Aisen PS, et al. Tracking pathophysiological processes in Alzheimer's disease: an updated hypothetical model of dynamic biomarkers. Lancet Neurol. 2013;12:207-16.

3. Guillozet AL, Weintraub S, Mash DC, Mesulam MM. Neurofibrillary tangles, amyloid, and memory in aging and mild cognitive impairment. Arch Neurol. 2003;60:729-36.

4. Jack CR Jr., Bennett DA, Blennow K, Carrillo MC, Dunn B, Haeberlein SB, et al. NIA-AA research framework: toward a biological definition of Alzheimer's disease. Alzheimers Dement. 2018;14:535-62.

5. Ottoy J, Niemantsverdriet E, Verhaeghe J, De Roeck E, Struyfs H, Somers C, et al. Association of short-term cognitive decline and MCI-to-AD dementia conversion with CSF, MRI, amyloid- and (18)F-FDG-PET imaging. Neuroimage Clin. 2019;22:101771.

6. Petzold A. Neurofilament phosphoforms: surrogate markers for axonal injury, degeneration and loss. J Neurol Sci. 2005;233: 183-98.

7. Trojanowski JQ, Walkenstein N, Lee VMY. Expression of neurofilament subunits in neurons of the central and peripheral nervous-system-an immunohistochemical study with monoclonalantibodies. J Neurosci. 1986;6:650-60.

8. Lycke JN, Karlsson JE, Andersen O, Rosengren LE. Neurofilament protein in cerebrospinal fluid: a potential marker of activity in multiple sclerosis. J Neurol Neurosur Psychitary. 1998;64: $402-4$.

9. Rosengren LE, Karlsson JE, Karlsson JO, Persson LI, Wikkelso C. Patients with amyotrophic lateral sclerosis and other neurodegenerative diseases have increased levels of neurofilament protein in CSF. J Neurochem. 1996;67:2013-8.

10. Scherling CS, Hall T, Berisha F, Klepac K, Karydas A, Coppola $\mathrm{G}$, et al. Cerebrospinal fluid neurofilament concentration reflects disease severity in frontotemporal degeneration. Ann Neurol. 2014;75:116-26.

11. Zetterberg H, Skillback T, Mattsson N, Trojanowski JQ, Portelius E, Shaw LM, et al. Association of cerebrospinal fluid neurofilament light concentration With Alzheimer disease progression. JAMA Neurol. 2016;73:60-7.

12. Bacioglu M, Maia LF, Preische O, Schelle J, Apel A, Kaeser SA, et al. Neurofilament light chain in blood and CSF as marker of disease progression in mouse models and in neurodegenerative diseases. Neuron. 2016;91:56-66.

13. Johnson EB, Byrne LM, Gregory S, Rodrigues FB, Blennow K, Durr A, et al. Neurofilament light protein in blood predicts regional atrophy in Huntington disease. Neurology. 2018;90: e717-e23.

14. Soylu-Kucharz R, Sandelius A, Sjogren M, Blennow K, Wild EJ, Zetterberg $\mathrm{H}$, et al. Neurofilament light protein in CSF and blood is associated with neurodegeneration and disease severity in Huntington's disease R6/2 mice. Sci Rep. 2017;7:14114.

15. Brureau A, Blanchard-Bregeon V, Pech C, Hamon S, Chaillou P, Guillemot JC, et al. NF-L in cerebrospinal fluid and serum is a biomarker of neuronal damage in an inducible mouse model of neurodegeneration. Neurobiol Dis. 2017;104:73-84.

16. Rohrer JD, Woollacott IOC, Dick KM, Brotherhood E, Gordon E, Fellows A, et al. Serum neurofilament light chain protein is a measure of disease intensity in frontotemporal dementia. Neurology. 2016;87:1329-36.

17. Mattsson N, Andreasson U, Zetterberg H, Blennow K, Alzheimer's Disease Neuroimaging I. Association of plasma neurofilament light with neurodegeneration in patients with Alzheimer disease. JAMA Neurol. 2017;74:557-66.

18. Mattsson N, Insel PS, Palmqvist S, Portelius E, Zetterberg H, Weiner $\mathrm{M}$, et al. Cerebrospinal fluid tau, neurogranin, and neurofilament light in Alzheimer's disease. EMBO Mol Med. 2016;8:1184-96.

19. Preische O, Schultz SA, Apel A, Kuhle J, Kaeser SA, Barro C, et al. Serum neurofilament dynamics predicts neurodegeneration and clinical progression in presymptomatic Alzheimer's disease. Nat Med. 2019;25:277-83.

20. Mattsson N, Cullen NC, Andreasson U, Zetterberg H, Blennow K. Association between longitudinal plasma neurofilament light and neurodegeneration in patients with Alzheimer disease. JAMA Neurol. 2019;76:791-9.

21. Buckner RL, Snyder AZ, Shannon BJ, LaRossa G, Sachs R, Fotenos AF, et al. Molecular, structural, and functional characterization of Alzheimer's disease: evidence for a relationship between default activity, amyloid, and memory. J Neurosci. 2005;25:7709-17.

22. Grothe MJ, Teipel SJ, Alzheimer's Disease, Neuroimaging I. Spatial patterns of atrophy, hypometabolism, and amyloid deposition in Alzheimer's disease correspond to dissociable functional brain networks. Hum Brain Mapp. 2016;37:35-53.

23. Seeley WW, Crawford RK, Zhou J, Miller BL, Greicius MD. Neurodegenerative diseases target large-scale human brain networks. Neuron. 2009;62:42-52.

24. Smith CD, Chebrolu H, Wekstein DR, Schmitt FA, Markesbery WR. Age and gender effects on human brain anatomy: a voxelbased morphometric study in healthy elderly. Neurobiol Aging 2007;28:1075-87.

25. Leon WC, Canneva F, Partridge V, Allard S, Ferretti MT, DeWilde A, et al. A novel transgenic rat model with a full Alzheimer's-like amyloid pathology displays pre-plaque intracellular amyloid-beta-associated cognitive impairment. J Alzheimers Dis. 2010;20:113-26.

26. Parent MJ, Zimmer ER, Shin M, Kang MS, Fonov VS, Mathieu A, et al. Multimodal imaging in rat model recapitulates Alzheimer's disease biomarkers abnormalities. J Neurosci. 2017;37: 12263-71.

27. Heggland I, Storkaas IS, Soligard HT, Kobro-Flatmoen A, Witter MP. Stereological estimation of neuron number and plaque load in the hippocampal region of a transgenic rat model of Alzheimer's disease. Eur J Neurosci. 2015;41:1245-62.

28. Sled JG, Zijdenbos AP, Evans AC. A nonparametric method for automatic correction of intensity nonuniformity in MRI data. IEEE Trans Med Imaging. 1998;17:87-97.

29. Smith SM. Fast robust automated brain extraction. Hum Brain Mapp. 2002;17:143-55.

30. Mazziotta JC, Toga AW, Evans A, Fox P, Lancaster J. A probabilistic atlas of the human brain: theory and rationale for its development. The International Consortium for Brain Mapping (ICBM). Neuroimage. 1995;2:89-101.

31. Fonov AE V, McKinstry R, Almli C, Collins. D. Unbiased nonlinear average age-appropriate brain templates from birth to adulthood. Neuroimage. 2009;47:S102.

32. Friedel M, van Eede MC, Pipitone J, Chakravarty MM, Lerch JP. Pydpiper: a flexible toolkit for constructing novel registration pipelines. Front Neuroinform. 2014;8:67.

33. Bittner T, Zetterberg H, Teunissen CE, Ostlund RE Jr., Militello $\mathrm{M}$, Andreasson U, et al. Technical performance of a novel, fully automated electrochemiluminescence immunoassay for the quantitation of beta-amyloid (1-42) in human cerebrospinal fluid. Alzheimers Dement. 2016;12:517-26.

34. Hansson O, Seibyl J, Stomrud E, Zetterberg H, Trojanowski JQ, Bittner T, et al. CSF biomarkers of Alzheimer's disease concord with amyloid-beta PET and predict clinical progression: A study 
of fully automated immunoassays in BioFINDER and ADNI cohorts. Alzheimers Dement. 2018;14:1470-81.

35. Gisslen M, Price RW, Andreasson U, Norgren N, Nilsson S, Hagberg L, et al. Plasma Concentration of the neurofilament light protein (NFL) is a biomarker of CNS injury in HIV infection: a cross-sectional study. EBioMedicine. 2016;3:135-40.

36. Mathotaarachchi S, Wang S, Shin M, Pascoal TA, Benedet AL, Kang MS, et al. VoxelStats: A MATLAB package for multimodal voxel-wise brain image analysis. Front Neuroinform. 2016;10:1-12.

37. Modvig S, Degn M, Horwitz H, Cramer SP, Larsson HB, Wanscher B, et al. Relationship between cerebrospinal fluid biomarkers for inflammation, demyelination and neurodegeneration in acute optic neuritis. PLoS ONE 2013;8:e77163.

38. Selkoe DJ, Hardy J. The amyloid hypothesis of Alzheimer's disease at 25 years. EMBO Mol Med. 2016;8:595-608.

39. Iulita MF, Allard S, Richter L, Munter LM, Ducatenzeiler A, Weise C, et al. Intracellular Abeta pathology and early cognitive impairments in a transgenic rat overexpressing human amyloid precursor protein: a multidimensional study. Acta Neuropathol Commun. 2014;2:61.

40. Sutphen CL, McCue L, Herries EM, Xiong C, Ladenson JH, Holtzman DM, et al. Longitudinal decreases in multiple cerebrospinal fluid biomarkers of neuronal injury in symptomatic late onset Alzheimer's disease. Alzheimers Dement. 2018;14:869-79.
41. Rojas JC, Karydas A, Bang J, Tsai RM, Blennow K, Liman V, et al. Plasma neurofilament light chain predicts progression in progressive supranuclear palsy. Ann Clin Transl Neurol. 2016;3:216-25.

42. Steinacker P, Blennow K, Halbgebauer S, Shi S, Ruf V, Oeckl P, et al. Neurofilaments in blood and CSF for diagnosis and prediction of onset in Creutzfeldt-Jakob disease. Sci Rep. 2016; 6:38737.

43. Jack CR Jr., Lowe VJ, Senjem ML, Weigand SD, Kemp BJ, Shiung MM, et al. 11C PiB and structural MRI provide complementary information in imaging of Alzheimer's disease and amnestic mild cognitive impairment. Brain 2008;131(Pt 3): 665-80.

44. Palmqvist S, Scholl M, Strandberg O, Mattsson N, Stomrud E, Zetterberg H, et al. Earliest accumulation of beta-amyloid occurs within the default-mode network and concurrently affects brain connectivity. Nat Commun. 2017;8:1214.

45. Welikovitch LA, Do Carmo S, Magloczky Z, Szocsics P, Loke J, Freund $\mathrm{T}$, et al. Evidence of intraneuronal Abeta accumulation preceding tau pathology in the entorhinal cortex. Acta Neuropathol. 2018;136:901-17.

46. Esparza TJ, Zhao H, Cirrito JR, Cairns NJ, Bateman RJ, Holtzman $\mathrm{DM}$, et al. Amyloid-beta oligomerization in Alzheimer dementia versus high-pathology controls. Ann Neurol. 2013;73:104-19.

\section{Affiliations}

\section{Min Su Kang $\mathbb{B}^{1,2,3} \cdot$ Arturo Aliaga Aliaga ${ }^{1,2,3} \cdot$ Monica Shin ${ }^{1,2} \cdot$ Sulantha Mathotaarachchi ${ }^{1,2}$. \\ Andrea L. Benedet $\mathbb{D}^{1,2} \cdot$ Tharick A. Pascoal ${ }^{1,2} \cdot$ Joseph Therriault $\mathbb{B}^{1,2} \cdot$ Mira Chamoun $^{1,2} \cdot$ Melissa Savard $^{1,2} \cdot$ Gabriel A. Devenyi $\mathbb{1}^{2,4} \cdot$ Axel Mathieu $^{2} \cdot$ M. Mallar Chakravarty ${ }^{2,4,5}$. Åsa Sandelius ${ }^{6} \cdot$ Kaj Blennow $\mathbb{B}^{6,7}$. Henrik Zetterberg ${ }^{6,7,8,9} \cdot$ Jean-Paul Soucy ${ }^{3} \cdot$ A. Claudio Cuello $\mathbb{1}^{10} \cdot$ Gassan Massarweh $^{3} \cdot$ Serge Gauthier $^{1,2,3}$. Pedro Rosa-Neto $\mathbb{1}^{1,2,3,4} \cdot$ Alzheimer's Disease Neuroimaging Initiative}

1 Translational Neuroimaging Laboratory, McGill University Research Centre for Studying in Aging, Montreal, QC, Canada

2 Cerebral Imaging Centre, Douglas Research Centre, Montreal, QC, Canada

3 McConnell Brain Imaging Centre, McGill University, Montreal, QC, Canada

4 Department of Psychiatry, McGill University, Montreal, QC, Canada

5 Department of Biomedical Engineering, McGill University, Montreal, QC, Canada
6 Department of Psychiatry and Neurochemistry, The Sahlgrenska Academy at the University of Gothenburg, Mölndal, Sweden

7 Clinical Neurochemistry Laboratory, Sahlgrenska University Hospital, Mölndal, Sweden

8 UK Dementia Research Institute at UCL, London, UK

9 Department of Neurodegenerative Disease, UCL Institute of Neurology, Queen Square, London, UK

10 Department of Pharmacology and Therapeutics, McGill University, Montreal, QC, Canada 